\title{
Design and Implementation of Extreme Weather and Climate Events Monitoring System
}

\author{
Liwei Liu*
}

College of Biology and the Environment, Nanjing Forestry University, Nanjing, JiangSu, China

\begin{abstract}
Status quo sudden environmental accidents occur frequently, in order to meet the needs of environmental risk management, designed weather and atmospheric environmental risk source monitoring system, the feature could easily cause a major environmental risk material incidents from the monitoring indicator system, monitoring distribution scheme, monitoring technology, monitoring data acquisition and transmission, monitoring and surveillance network database to build six aspects of information platform to study climate change and build sets very day climate for continuous on-line source of one of the environmental risks of real-time monitoring system, the effective implementation of environmental risk sources management and accident prevention.
\end{abstract}

Keywords: Extreme weather, monitoring system, system design, weather events.

\section{INTRODUCTION}

Monitoring is the use of television camera equipment, audio transmission equipment and processing equipment, as required by law to observe, to acquire and process-specific sites, specific personnel information, mastered by comprehensive technical activities and events of interest to monitor the development of personnel. Nowadays, with the rapid development of computer technology and digital image processing technology, monitoring technology has been widely applied to the security, industrial production, transportation and other fields, has become a social life, a very important part [1].

The actual effect of image quality and monitoring system monitoring system is closely related to the judgment scene monitoring the situation, the high-resolution, high-definition image monitoring is particularly important. But in real life, images collected by the imaging system are more or less there will be degradation, and even the loss of image information. There are many causes of image degradation, nonlinear, image defocus blur include photoelectric sensors, the impact of film recording material particles, atmospheric turbulence and scattering affect the optical lens, the scene with the relative motion of the camera and so on. Usually blurred image degradation, image detail information is lost, it is difficult to extract useful information. So, in this situation, image enhancement technology has been rapid development [2].

Monitoring system for enhanced collection, you need to use a variety of image enhancement techniques and mathematical methods changing means to improve image sharpness and contrast, the original blurred, cannot distinguish between the original image processing has become clear, clear, contains a large number of useful image information, so as to achieve outstanding image feature information, the purpose of improving the image may recognizable. It can be seen clearly extreme weather and climate events processing conditions under surveillance images have important practical significance.

\section{MONITORING SYSTEM DESIGN}

Emergency Monitoring capacity building, including monitoring systems and drinking water sources in the security information management system consists of three parts of a monitoring system and monitoring capacity building should be integrated in accordance with the rational distribution of resources focused information sharing arrangements for the construction of the principles of monitoring system, including the establishment of appropriate means of on-line monitoring, through real-time online monitoring of water quality parameters to control part of, and should establish a water quality monitoring instrument according to sources corresponding features, including water toxicity monitoring instrument line biological agents monitor various types of water quality monitoring and fast line monitor routine monitoring instruments through the establishment of monitoring instruments water in security monitoring coordination mechanisms [3], coordination and management of water sources to water quality monitoring, sharing and dissemination of information resources to establish and improve the water quality monitoring system and information system, the basic realization of real-time online monitoring.

\subsection{Coordinate the Monitoring System Set}

\subsubsection{Coordinate Convention}

One digital image $f(x, y)$ is sampled to generate $M$ rows and $\mathrm{N}$ columns, the value of the coordinates $(\mathrm{x}, \mathrm{y})$ becomes 
discrete, so that the origin coordinate values $(x, y)=(0,0)$, the first line of a next coordinate $(x, y)=(0,1)$, a first column of a coordinate $(x, y)=(0,2)$, and so on (Fig. 1) [3].

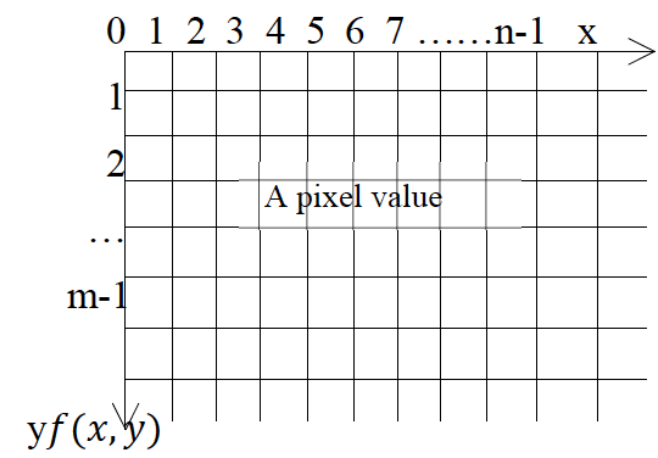

Fig. (1). Coordinate convention.

\subsubsection{Function Matrix}

The coordinate convention, the digitized result is a matrix representation, in the computer to two-dimensional function, expressed in matrix form, record. An image $\mathrm{f}(\mathrm{x}, \mathrm{y})$ can be expressed as below formula [4].

$$
f(x, y)=\left[\begin{array}{cccc}
\mathrm{f}(0,0) & \mathrm{f}(0,1) & \ldots & \mathrm{f}(0, \mathrm{~N}-1) \\
\mathrm{f}(1,0) & \mathrm{f}(1,1) & \ldots & \mathrm{f}(1, \mathrm{~N}-1) \\
\ldots & \ldots & \ldots & \ldots \\
\mathrm{f}(\mathrm{M}, 0) & \mathrm{f}(\mathrm{M}, 1) & \ldots & \mathrm{f}(\mathrm{M}-1, \mathrm{~N}-1)
\end{array}\right]
$$

\subsection{Monitoring System Image Processing Method Clear}

\subsubsection{Histogram Linearization Method}

Histogram linearization method is a simple and practical method of calculation [5]. Histogram linearization method [4] for processing is the luminance gradation image size of each pixel. Histogram of the digital image in gray level $[0,1]$ is a discrete function of the range $\mathrm{A}$, where $\mathrm{r}$ is the $\mathrm{k}$-th gray levels, $\mathrm{L}$ is the number of pixels at this level gray. That often divided by the total number of pixels of the image for each value, resulting normalized histogram. If $r$ is normalized to the interval $[0,1]$, then when $r=0$, when black, $r=1$ for white. For the transformation of the form $\mathrm{r}$ :

$$
\mathrm{s}=\mathrm{T}(\mathrm{r}), 0 \leq r \leq 1
$$

For the original image, each pixel value to generate a gray scale value $\mathrm{r}$. $\mathrm{T}(\mathrm{r})$ satisfies the condition:

\section{a. In $0 \leq \mathrm{r} \leq 1$ as a single value and monotonically. \\ b. When $0 \leq \mathrm{r} \leq 1$ time, $0 \leq \mathrm{T}(\mathrm{r}) \leq 1$.}

When the image is reversed certain gray value, grayscale inversion occurs, the use of inverse transform $r=T^{\wedge}(-1)(s)$ to be resolved.

A grayscale image, but the interval $[0,1]$ random variables, that is, using the probability density function (PDF) to obtain:

$$
P_{s}(s)=P_{r}(r)\left|\frac{d r}{d s}\right|
$$

Wherein, $\mathrm{P}_{-} \mathrm{r}$ with $\mathrm{P} \_\mathrm{s}$ representing $\mathrm{r}$, s probability density function. Therefore, changing the variable $\mathrm{s}$ is deter- mined by the probability density function of the input image and grayscale PDF conversion function selected.

Given the transformation function $\mathrm{T}(\mathrm{r})$, into the above equation to get $\mathrm{P}_{-} \mathrm{s}$ (s). Derivative according to the points that define the upper limit of the integral value, obtained $\mathrm{dr} /$ ds $=P_{-} r(r)$, in place of the above yields:

$$
P_{s}(s)=P_{r}(r)\left|\frac{d r}{d s}\right|=1 \quad 0 \leq r \leq 1
$$

In the image of the moving average process, the probability of an image gray value rand $\mathrm{k}$ appears approximately:

$$
P_{r}\left(r_{k}\right)=\left|\frac{n_{r}}{n}\right| \quad k=0,1,2,3,4 \cdots \cdots, L-1
$$

Wherein $\mathrm{n}$ is the sum of the pixel, $\mathrm{r}_{-} \mathrm{k}$ is grayscale $\mathrm{r}_{-} \mathrm{k}$, the number of pixels, $\mathrm{L}$ is the total number of gray level. Here $P_{-} r$ ( $\left.r_{-} k\right)$ gives an estimate of the probability of occurrence $r_{-} k$, so the original image histogram provides the distribution of gray values, that is to say on a new original grayscale images were gray distribution. In order to get clear of the original image, the use of the algorithm is to expand the distribution of gray levels of the image, so that the visual image on the structured and clear, this is actually the equivalent of increasing the contrast of each pixel in the image. It also introduced a interpolation and a non-overlapping histogram linearization method [6]. Histogram linear algorithm is the application of this method, the original image is converted into a uniformly distributed histogram expand, by a mathematical algorithm to make the histogram of the processed image becomes clear hierarchy, each gradation level has this same frequency number, so that each gradation level also has a balanced probability distribution, so that the original image is processed by histogram after linearization algorithm will transform clearer.

For discrete patterns can transform:

$$
s_{k}=T\left(r_{k}\right)=\sum_{j=0}^{k} p_{r}\left(r_{j}\right)=\sum_{j=0}^{k} \frac{n_{j}}{n}
$$

Accordingly, the image processed by the above formula for the gray level is mapped to each pixel on the output image $r_{-} k$ grayscale $s_{-} k$ display. As mentioned above, $r_{-} k$ function $\mathrm{P}_{\mathrm{r}} \mathrm{r}\left(\mathrm{r}_{-} \mathrm{k}\right)$ curve is called a histogram. Conversion formula given by (mapped) is called a histogram linearization or histogram equalization.

Histogram linearization is a popular image enhancement method, it belongs to the non-linear spatial domain single point enhanced digital image processing method, the histogram linearization algorithm operating and programming time is short, fast image post-processing to achieve. When the image gray value is distribution within a specific range, the processed image is more clarity. However, due to the discrete gradation values, the image portion liberalized dynamic enhancement, its essence expanding the pixel gray values between the quantization interval, the number of gray rather than quantization, on the contrary, the distribution becomes small after linearization, for gradation frequency smaller areas, the contrast is reduced, or perhaps disappear, 
explained by the theory that the above histogram linear high degree of sensitivity to noise [7].

\subsubsection{Homomorphism Filtering Processing Method}

A homomorphism filtering is the grey value as a product of the intensity of illumination and reflectance, due to the intensity of illumination is relatively small, can be regarded as low frequency component of the image and the reflectivity is high frequency components. Through processing illumination and reflectance grey value of pixels, respectively, the influence of shadow detail characteristics.

In extreme weather and climate events, pollutants have lower liquidity, range relative to the air space is little, to be stable distribution, the extreme weather and climate events under the degradation of image processing, we are interested in the part of the gray level will be very dark, every detail in the image clarity is low, there is no way to identify, the average gray level transform is no good [8]. And homomorphism filtering of images belongs to the category of image frequency domain processing, effect for image gray scale adjustment, eliminate the problem of non-uniform illumination on the image, enhance the details of the dark area, and not lose the higher brightness area details, this process is also based on the enhanced contrast method.

Homomorphism filtering is based on the illumination reflection model in the frequency domain, it enhance the visibility of the after image processing, improve the image resolution [5]. General natural scenery images can be made of illumination function, and the reflection function of the product, according to describe the scene lighting, has nothing to do with scenery, contains details scenery, has nothing to do with the lighting. Get this formula:

$$
\begin{aligned}
& f(\mathrm{x}, \mathrm{y})=f_{i}(x, y) * f_{r}(x, y) \\
& 0<f_{i}(x, y)<\infty, 0<f_{r}(x, y)<1
\end{aligned}
$$

Incident light to light, reflected light show its scenery properties, so the scenery brightness depends on the reflected light. Radiation uniformity of incident light in space, the space changes basically remain unchanged, the reflected light is because each object giving out light reflection to the scenery itself after the shape of the volume of each part is different, lead to reflect light range is larger. Therefore, in the spatial frequency domain, incident light have the low frequency part, anyway, the reflected light to occupy the high frequency part of wider. So we have to do to reflect light and reflected light of different image processing methods. Deformation by formula:

$$
\begin{array}{r}
\ln f(x, y)=\ln \left[f_{i}(x, y) * f_{r}(x, y)\right] \\
=\ln f_{i}(x, y)+\ln f_{r}(x, y)
\end{array}
$$

Make in the spatial domain combined relationship, to make Fourier transform formula to get:

$$
\begin{array}{r}
F_{\ln }(u, v)=F\left[\ln f_{i}(x, y)+\ln f_{r}(x, y)\right] \\
=F_{i, l n}(u, v)+F_{r, l n}(u, v)
\end{array}
$$

Type, as the lighting function on the space changes slowly, the spectrum characteristic is concentrated in the low frequency part, as the reflection function spectrum is concentrated in the high frequency part of the reflection function describing scenery, reflect the detail of the image content, its frequency in the high frequency region. If the uneven illumination of the image, the image of each part on the average brightness there will be ups and downs, the dark area of the image detail is hard to convenient, need to deal with this is not uniformity. Can compress the grayscale range of lighting function, reduce illume function in frequency domain, enhanced reflection spectrum part function, enhance its contrast. The dark part of the final image details highlight [9]. By on the transfer function $\mathrm{H}(\mathrm{u}, \mathrm{v})$ (homomorphism filter), low frequency part of the compression, and the high frequency part release. Filter transfer function $\mathrm{H}(\mathrm{u}, \mathrm{v})$ after the stand or fall of choice for processing image effect is very important [3].

Homomorphism filtering function formula is expressed as:

$$
\begin{aligned}
G_{l \mathrm{n}}(u, v)=F_{l n}(u, v) * \mathrm{H}(\mathrm{u}, \mathrm{v}) & \\
& =F_{i, l n}(u, v) * \mathrm{H}(\mathrm{u}, \mathrm{v})+F_{r, l n}(u, v) \\
& * \mathrm{H}(\mathrm{u}, \mathrm{v})=G_{i, l n}(u, v)+G_{r, l n}(u, v)
\end{aligned}
$$

Its Fourier inverse transformation, get the corresponding spatial domain formula:

$$
F^{-1}\left\{G_{l n}(u, v)\right\}=F^{-1}\left\{G_{i, l n}(u, v)\right\}+F^{-1}\left\{G_{r, l n}(u, v)\right\}
$$

Finally, the formula of index after enhanced image formula:

$$
\mathrm{g}(\mathrm{x}, \mathrm{y})=\exp \left\{F^{-1}\left\{G_{l n}(u, v)\right]\right\}
$$

We can find that according to the characteristics of different objects in the image, you can switch to a different $\mathrm{H}$ (u, v) homomorphism filter, achieve satisfactory effect. Details in the original image contrast, resolution of image after image can use processing of homomorphism filter screen brightness is evener, detail improved remarkably.

\subsection{Monitoring System Image Processing Method}

In the color image processing can be divided into two categories; one is a full-color image processing, and the other is a pseudo-color image processing. With a full-color image acquisition is device in the first category, such as color television, scanners. The second category for single brightness with a color can give a certain range.

\subsubsection{Color Model}

In digital image processing more generic model for RGB color model. Mainly because the first color image acquisition machine preclude RGB data are set, the color property on machine reaction is RGB data. In the RGB model space, each color is composed of three components R Red, G Green, B blue color formation of different proportions [7]. In Cartesian coordinates as follows:

Fig. (2) shows that according to R, G, B three points located on three corners, magenta, cyan, yellow on the other three corners, black at the origin of the coordinate axes, then the white cube body diagonal, overall color distribution in the body diagonal, all R, G, B of the range of values in $0 \leq x \leq 1$, by model, all images are composed of three image surface components, each component represents a primary image plane, the image sensor, when received, will form a three color overlay a color image [9]. 


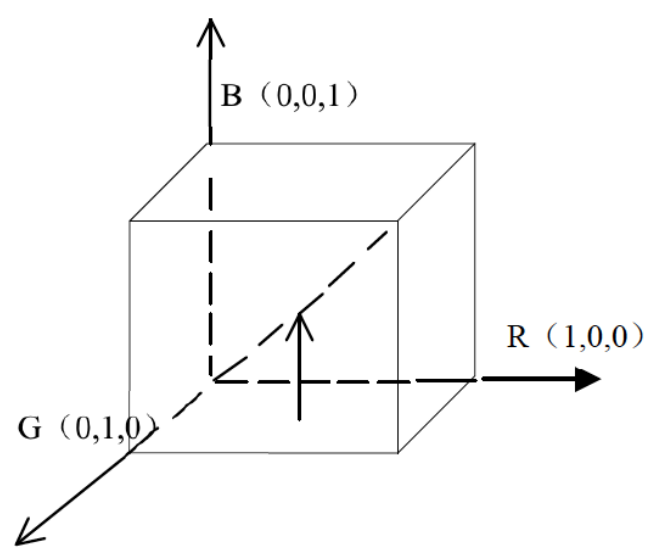

Fig. (2). RGB color cube.

\subsubsection{Color Image Processing}

The full color image and an artwork like by natural color images, full color image processing is a kind of can processing each component of the image, respectively, and then synthesis processing component of the image formation of color images, another kind is to deal directly with each color pixels to get new image. Image acquisition machine is commonly used in our life is common RGB mode, so in dealing with color degradation image is also is processed in the synthesis of the original image in RGB space in new color images, this paper mentioned homomorphic filtering restoration and Retinex recovery is color image processing [10].

For color image processing method, mainly according to the color of each pixel in the image to adjust the overall image of color visual effect, not from human eyes visual approach, although some aspects of the image detail better treatment, but the overall effect is poor, in the treatment of the noise at the same time also back some scenes details in images [1]. So in order to get the better of this part of processing, Retinex processing, put forward his theory and algorithm of color distortion of the image effect is well improved. Its method is based on people eye visual perception of color information, using the perception of color effect on the original haze image color enhancement processing.

\subsubsection{The Development of the Retinex Method}

According to the theory of color vision, perception of the human eye to color the frequency of the degree mainly depends on the wavelength of light, the wavelength at different frequencies have different color, so the space that we see the color of the scenery, is due to the different frequency of light waves hit the above objects into the human feeling of color. This theory can well explain the atmosphere all the phenomenon of color. HLand found some color phenomenon is a traditional theory fails to explain, such as color constancy [11]. This kind of phenomenon is the light of the change in the situation different people on the same object or different judging color is different, can say as a psychological dependence, the judgment of this color is a slight change in a certain range, can approximate expression for the phenomenon of color constancy.

Land thinks people react to the visual signals to judge, in addition to the light illuminate objects give to some small color difference caused by uneven factors, keep basic attrib- ute information objects. Through the senses into the brain to respond, and finally into the human visual system in 1977, Edwin $\mathrm{J}$ publishedthe theory and put forward for the first time, also called retina cortex system theory [10]. Theory of illumination change is slow, a slight change in color can be thought of as a smooth illumination, surface caused by the change is a mutated form, resolution object surface micro change color, after color and reflection to the intensity of illumination changes and surface distinguish, makes the surface color constant. Edwin. H, the Land that the human eye to brightness has nothing to do with illumination itself, from a conclusion, each retina system so that the human eye to color all has the ability to judge alone, for high band, respectively when light wave propagation in the band, short wave period of independent reaction, set up three separate scene, rendering the three bands of independent color additive, make the image present different color.

\subsubsection{Color Constancy}

People eye visual perception of color, when the light intensity of illumination changes, can maintain basic unchanged, show the color constancy. Color constancy is not due to external conditions, under the influence of maintain inner a propensity for color. That is for the same object at different bearing surface will result in different intensity of illumination of the reflected light frequency, small changes to the color of light anyway cause can make a different degree of decision, but one of the object surface color the small changes or think reasonable, did not respond to their own judgment is constant, which argues for an object's surface without color changes [11].

Eye of the observed object color is not completely corresponding with wavelength, intensity, etc. Perception of color is derived from the outside factors, judgment without relying on outside factors. At present many researchers use the scientific analysis are not well explained. Many scientists according to their hypothesis createdtheir own model. The earliest research has two kinds, the first kind is put forward by Helmholtz, it is a kind of "rational judgment is established on the unconscious feeling". The second is the black forest put forward the concept of "color" of memory.

\section{SYSTEM MONITORING IMAGE CLARITY EVALUATION}

With the rapid development of science and technology, digital image processing occupies a more and more important in our life. As the scientific research work of digital image processing is one of the degraded image processing methods, means, tools, its practicability is strong, wide coverage range [12]. Handling of the degraded images also has its evaluation standard and methods. For digital images, for example, machine vision distance set equipment parameter correction; need to use the image quality evaluation. After processing the quality of image restoration is clear, also want to use the image quality evaluation, etc. At present, the digital image processing after image quality evaluation method mainly has two kinds of methods, one kind is visual subjective image quality assessment method, and another kind is the objective image quality evaluation parameters. 
Table 1. Visual subjective image quality evaluation.

\begin{tabular}{|c|c|c|}
\hline Rank & Absolute Measurement Scale & The Relative Measurement Scale \\
\hline \hline 1 & Best & The best in a group \\
\hline 2 & Better & The better in a group \\
\hline 3 & Good & Bad in the group \\
\hline 4 & Bad & The worse in the group \\
\hline 5 & Worse & The worst in the group \\
\hline 6 & Worst &
\end{tabular}

\subsection{Visual Subjective Image Quality Assessment Method}

Visual subjective image quality assessment method, is to make the evaluation measure of observer based on a series of rules or according to your own experience, the image of the test according to the best visual effects gives its judgment, the quality of the images and the quality of image, based on the participation of image evaluation observer is given on the data are calculated, the results obtained the final image is the subjective image quality evaluation [4].

There are two main types of subjective evaluation of the image measurement scale, the first for the absolute scale, is the relative dimension [13], (as shown in Table 1). Although this method has good intuitive feelings quickly reflect the quality of the image, but unable to use conventional theoretical calculation parameters description of the image. In practice, the visual subjective image quality assessment method is limited by a lot.

\subsection{Objective Image Quality Assessment Method of Pa- rameters}

At present, there is no unified image quality evaluation system and evaluation method, but according to starting from the basic properties and parameters of an image, the image of a reasonable objective evaluation. According to the following basic parameters were compared.

\subsubsection{Clarity}

Definition refers to each detail shadow lines in the image and its degree of clear boundaries. At present, the quality of digital image processing of clarity evaluation method has two kinds, one kind is the airspace parameter method, the other is a frequency domain method. For airspace evaluation algorithm of parameter method is simple, fast processing speed, a slight change of image is not affected [13]. Frequency domain method, processing speed slow, complex algorithm, the image of the tiny had a greater influence on the transformation, shall not apply to the program automatically.

For clarity describes calculation formula is expressed as:

$$
\mathrm{DEF}=\frac{\sum_{\mathrm{i}=1}^{\mathrm{mxn}} \sum_{\mathrm{a}=1}^{8}\left|\frac{\mathrm{df}}{\mathrm{dx}}\right|}{\mathrm{m} * \mathrm{n}}
$$

Type, $\mathrm{m}, \mathrm{n}$ as the image size, $\mathrm{df}$ for grayscale values change, the distance between $\mathrm{dx}$ for each pixel. Mainly in about eight points around each pixel, the superposition of difference sum. Formula, its near the main measuring around each pixel in the image grey value diffusion degree, the larger the diffusion, its value, the greater the image resolution is higher, is proportional to the relationship.

\subsubsection{Contrast Ratio}

Contrast is an image of the clear degree of physical quantities, is on the edge of the image of the grain and the floorboard of the clear degree. Contrast the co-occurrence matrix, the deeper the degree of grain, the greater the contrast [6]. Grain is proportional to the contrast relationship. Its computation formula is expressed as:

$$
f=\sum_{n=0}^{L-1} n^{2} \sum_{i=0}^{L-1} \sum_{j=0}^{L-1} p(i, j) n=|i-j|
$$

\subsubsection{Entropy}

The size of the bearing information entropy is digital image, image texture, the more complex the image hosting the more the amount of information [9], so the more. Its computation formula is expressed as:

$$
f=-\sum_{n=0}^{L-1} \sum_{i=0}^{L-1} p(i, j) \log p(i, j)
$$

When the gray value of each pixel in the image at the same time, its data value is the largest, with should be for a single color image, the minimum value data.

The above important standard for objective evaluation of digital image processing, image provides objective in to deal with the noise data support, evaluation and analysis on the image noise is also a kind of important evidence to support the after image processing.

\section{CONCLUSION}

Knowledge of this chapter mainly introduces the basic theory of digital image, digital image quality evaluation method and the processed image is degraded visualization standard evaluation parameters. Detailed lists several kinds of common digital image processing, image processing enhancement methods, such as histogram linearization method of homomorphic filtering processing method, color map image processing method and the Retinex treatment principle. In order to solve and improve these problems, we should 
further in digital image processing by establishing the mathematical and physical model on the basis of studying its haze mechanism of image degradation, and then taken to deal with the analysis of extreme weather and climate events.

The design of effective monitoring of abrupt climate events, providing pollution concentration of decision-making departments deal with the accident, the distribution of the impact, extent and development trend of information occurred and be able to analyze the main factors of the accident, the effective implementation of the environmental risk management and source accident prevention.

\section{CONFLICT OF INTEREST}

The author confirms that this article content has no conflict of interest.

\section{ACKNOWLEDGEMENTS}

Declared none.

\section{REFERENCES}

[1] J. G. Qin, and S. H. Han, "Neurocognitive mechanisms, underlying identification of environmental risks," Neuropsychologia, no. 47, pp. 397-405, 2009. (In Chinese)

[2] X. L. Wang, "Evaluation of Wetland Biodiversity", China EcoAgriculture, vol. 36, pp. 6-17, 2010. (In Chinese)
[3] X.Y. Zhou, and W. Xu, "Chinese Coastal Wetlands", China Forestry Publishing House, vol. 35, pp. 24-28, 2011. (In Chinese)

[4] D.S. Wang, and H.W. Tan, "Qilihai Wetland Ecological Water Demand", Hebei Agricultural Science and Technology, vol. 4, pp. 298-310, 2010. (In Chinese)

[5] Q.S. Liu, "Wetlands and wetland protection", China Environmental Science Press, pp. 19-31, 2012. (In Chinese)

[6] S. Zhu, "Process and Environmental Effect Qilihai lagoon wetland evolution", Wetland Science, vol. 40, pp. 135-136, 2013. (In Chinese)

[7] Y. Hui, C.C. Li, and H.H. Zhao, "The climatechange and its effect on the water environment in the Hulun Lakewet land", Journal of Glaciology and Geocryology, vol. 29, pp. 795-801, 2007. (In Chinese)

[8] Y. W. Wen, H.X. Lin, and X.Zh. Lan, "Impacts of climatechange on Honghu Lakewet lands", Resources and Environment in the Yangtze Badin, vol. 6, pp. 20-22, 2003. (In Chinese)

[9] Y.H. Wang, and W.P. Cao, "Advances in the ecohydrology research", Sciatica Geographical Silica, vol. 35, pp. 67-69, 2009. (In Chinese)

[10] J. Ch. Zhong, and F.S. Liu, "Influence of global climate change on wetlands", lake science, vol. 21, pp. 335-344, 2011. (In Chinese)

[11] Q.K. Pan, Y. Cai, and J.J. Liang, "Increasing flood risk and wetland losses due to global sea-level rise: regional and global analyses," Environmental Protection and Prevention, vol. 13, pp. 27-34, 2012. (In Chinese)

[12] X.S. Wang, and X.J. Liang, "Wetlands carbon cycle and its significance in global change," Jilin Science and Technology Press, pp. 54-65, 2011. (In Chinese)

[13] Z.W. Wang, R.F. Liu, and Zh.J. Li, "Qilihai wetland ecosystem degradation and restoration", Soil and Water Conservation, vol. 12, pp. 244-248, 2005. (In Chinese)

(C) Liwei Liu; Licensee Bentham Open.

This is an open access article licensed under the terms of the (https://creativecommons.org/licenses/by/4.0/legalcode), which permits unrestricted, noncommercial use, distribution and reproduction in any medium, provided the work is properly cited. 